\title{
Semi-supine Exercise Stress Echocardiography in Children and Adolescents: Feasibility and Safety
}

\author{
P. Ciliberti • I. McLeod · F. Cairello • \\ J. P. Kaski · M. Fenton · A. Giardini • \\ J. Marek
}

Received: 30 July 2014/ Accepted: 31 October 2014/Published online: 20 November 2014

(C) The Author(s) 2014. This article is published with open access at Springerlink.com

\begin{abstract}
Although exercise stress echocardiography (ESE) is a well-validated technique in adult population, its use in children is quite limited. We aimed to assess the feasibility, the safety and the reproducibility of ESE, using on-line scanning in semi-supine cyclo-ergometer protocol in a large pediatric population. Between July 2008 and January 2013, 42 patients (mean age $14 \pm 3$ ) were evaluated with a bicycle ESE performing 50 studies. ESE was successfully performed and well tolerated by all patients. None of the patients presented with adverse effects of stress-induced ischemia. HR was $82 \pm 13$ at rest, and $153 \pm 19.1$ during peak exercise. Among 544 views analyzed for grading of image quality, the visualization was optimal in 473 (87\%), suboptimal in 39, and inadequate in $32(6 \%) .37$ tests were performed in patients with congenital or acquired coronary abnormality. Regional wall motion abnormalities (RWMA) were revealed in nine cases $(24 \%)$. The agreement between the two different observers showed a $K$ index of 0.7276 (95\% CI $0.6497-0.8055$ ) for the image quality and a $K$ index of 0.5125 (95\% CI 0.4782-0.5468) for the RWMA analysis. Among ten patients with hypertrophic cardiomyopathy, we were able
\end{abstract}

P. Ciliberti, I. McLeod, F. Cairello, J. P. Kaski, M. Fenton, A. Giardini, J. Marek takes responsibility for all aspects of the reliability and freedom from bias of the data presented and their discussed interpretation.

P. Ciliberti - I. McLeod - F. Cairello - J. P. Kaski - M. Fenton ·

A. Giardini · J. Marek ( $\square)$

Great Ormond Street Hospital for Children, London WC1N 3JH,

UK

e-mail: Jan.Marek@gosh.nhs.uk

J. P. Kaski · M. Fenton · A. Giardini · J. Marek Institute of Cardiovascular Sciences, University College London, London, UK to demonstrate the new comparison of significant left ventricular outflow tract gradient $(\geq 30 \mathrm{mmHg})$ during exercise in three patients $(30 \%)$. Bicycle stress echocardiography performed by on-line scanning during exercise is a feasible, safe, and reproducible modality in children. Further data to assess its diagnostic accuracy are, however, needed. Stress echocardiography provides a dynamic assessment of the myocardial structure and function under conditions of physiologic or pharmacologic stress.

Keywords Echocardiography $\cdot$ Exercise test $\cdot$ Pediatric cardiology $\cdot$ Coronary artery disease $\cdot$ Hypertrophic cardiomyopathy

\section{Background}

Stress-induced abnormalities of ventricular wall motion in patients with ischemic heart disease were recognized in 1979 [25]. Since then, stress echocardiography has been extensively applied in patients with coronary artery disease (CAD), and it has become a well-accepted modality for diagnosis, risk stratification, and prognostication in this setting [13, 39]. Its use has subsequently been extended to other clinical settings such as patients with hypertrophic $[32,40,43]$ or dilated cardiomyopathy, valvar heart disease $[7,44,45]$,pulmonary hypertension $[8,21,22]$ and recently for selecting potential responders to cardiac resynchronization therapy $[33,42]$ and for the selection of donor hearts for cardiac transplantation [6].

The stressor agent can be pharmacologic or physiologic. Dobutamine is the drug most widely used, although adenosine and dipyridamole are also commonly adopted. Physical exercise is the ideal stressor, being able also to provide information about symptoms, exercise capacity, and 
hemodynamic response to exertion [17, 39]. Exercise stress echocardiography (ESE) is most commonly performed on the treadmill, using similar protocols adopted for exercise ECG, or with a bicycle in either upright or supine position.

Despite wide experience in adults, stress echocardiography in the pediatric age group is rarely used [27, 41] and primarily reserved for patients with Kawasaki disease [28, $37]$ and pediatric transplant recipients [12, 30]. A small number of reports have been published in patients with congenital heart diseases [11, 23].

The major disadvantage of ESE in pediatric age is the precipitous drop in heart rate after peak exercise compared to adults [37]. In addition, some concerns exist about the image quality due to the higher heart rate in children, and the method's reproducibility in this setting has not previously been assessed. Bicycle exercise in the semi-supine position, compared to treadmill, offers the advantage that images can be obtained during all stages of exercise (including peak) in real time, avoiding the rapid heart rate drop. Instead of missing the peak heart rate due to its drop from erect position to a bed for imaging, recumbent position and continuous scanning should potentially allow better detection of ischemia and time for higher quality of images. Our aim hence was to assess the feasibility, safety, and reproducibility of dynamic ESE using "on-line" scanning in semi-supine cyclo-ergometer protocol in a wide spectrum of children with different types of disease.

\section{Methods}

\section{Patients}

Data of all patients undergoing a bicycle exercise echocardiography at the echocardiography unit of the Great Ormond Street Hospital for Children between July 2008 and January 2013 were retrospectively analyzed.

\section{Exercise Echocardiography Protocol}

All examinations were performed according to our standardized institutional laboratory protocol. A symptomlimited exercise test was conducted on a semi-supine bicycle ergometer (Lode Medical Technology, Groningen, The Netherlands) using a continuous incremental bicycle protocol with a work rate increment between 5 and $20 \mathrm{~W} /$ min according to gender and weight. All patients were given the opportunity to familiarize themselves with the cycle ergometer, and the procedure was discussed with the patient and parent/guardian prior to the test starting. Blood pressure measurement and clinical review preceded all tests. To be able to perform the test, all the children were at least $120 \mathrm{~cm}$ in height and above 7 years of age.
Echocardiography images were assessed at baseline, at low work load exercise at peak exercise, and during recovery, using a GE VIVID 7(GE Healthcare, Milwaukee, WI, with $4 \times 4$ Stress protocol adapted to our protocol). Peak exercise was defined as the maximum level of performance achieved before patient's exhaustion. The low work load images were acquired after an increase in heart rate by $20 \%$, during a level of workout that the patient was still comfortable with. All scans were performed with harmonic fusion imaging using a M4S probe. For the assessment of CAD, according to our clinical laboratory protocol, images of the left ventricle (LV) at each exercise stage were obtained from apical 4-chamber, 2-chamber, parasternal long-axis view, and parasternal short-axis view at the levels papillary muscles. All images were recorded digitally and analyzed off-line by the same observer (JM) using a 16-segments, 5-point scale model of the left ventricle according to the recommendations of the European Association of Echocardiography [29].

Studies performed for reasons other than assessment of CAD did not follow this 16-segment protocol at each stage, and instead, a more focused assessment was performed in line with the reason for referral. Specifically, for patients with HCM, an assessment of the presence and extent of systolic anterior motion of the mitral valve (SAM), presence and severity of mitral regurgitation, and left ventricular outflow tract gradient was performed, according to previously published techniques in adults [43].

The image quality was assessed for each acquired view on a three-point scale: 1 . complete endocardial definition and wall thickening; 2 . inadequate visualization of one or two LV segments; 3. inadequate visualization of three or more LV segments

\section{Coronary Angiography and Cardiac MRI}

When coronary angiography or cardiac MRI was performed within 6 months from the ESE (without any significant clinical event during this period), these exams were used for comparison with the ESE results.

\section{Interobserver Variability}

A representative sample $(29,78 \%)$ of the scans performed for evaluation of coronary artery disease was independently and blindly reviewed off-line by two different observers (PC and IM), not aware of patients' medical history, for assessment of image quality and presence of regional wall motion abnormalities (RWMA). For the RWMA analysis, the agreement between the two observers was tested for each of the 16 segments at every stage of the protocol. For the image quality, the agreement was tested for each acquired view. 
Table 1 Main clinical characteristics and results of the entire cohort of test performed

\begin{tabular}{ll}
\hline & Total, $n=50$ \\
\hline Male sex, $n(\%)$ & $28(56 \%)$ \\
Age at exam (year) & $14 \pm 3$ \\
BSA & $1.4 \pm 0.5$ \\
Indication, $n(\%)$ & \\
Coronary artery disease detection & $37(74 \%)$ \\
HCM & $10(20 \%)$ \\
Other & $3(6 \%)$ \\
Patients on medication, $n(\%)$ & $11(22 \%)$ \\
Beta blockers & $7(14 \%)$ \\
Calcium channel blockers & $4(8 \%)$ \\
ACE inhibitor & $2(4 \%)$ \\
Termination due to muscular exhaustion, $n(\%)$ & $50(100 \%)$ \\
ECG abnormalities during stress, $n(\%)$ & $5(10 \%)$ \\
Arrhythmia, $n(\%)$ & $0(0 \%)$ \\
Symptoms & $2(4 \%)$ \\
Rest HR (bpm) & $82 \pm 13.6$ \\
Peak HR (bpm) & $153.4 \pm 19.7$ \\
\hline
\end{tabular}

\section{Statistical Analysis}

All continuous variables were presented as mean with standard deviation. Categorical variables were reported as number and percentage. The weighted Cohen's Kappa test was used to assess the agreement between the two observers. A $K$ value $<0.20$ was considered to reflect poor reproducibility, $0.21-0.40$ fair, $0.41-0.60$ moderate, 0.61-0.80 good, and 0.81-1.00 was considered suggestive of a very good reproducibility [2].

\section{Results}

Patients

Forty-two (42) patients (23 males, $55 \%$, mean age $14 \pm 3$ years, median age 14.5 , range 6-22) were enrolled in the study, and 50 tests were performed. Six patients underwent two studies, and one patient underwent three studies. No differences were found between different studies performed by the same subject. Thirty-seven (74\%) tests were executed for assessment of coronary artery disease, ten $(20 \%)$ tests were for assessing LV outflow tract gradient during exercise in patients affected by hypertrophic cardiomyopathy (HCM), and three tests ( 1 patient affected by dilated cardiomyopathy, 1 patient affected by pulmonary hypertension, and 1 patient affected by dynamic LV outflow tract obstruction) were accomplished for different reasons. The main demographic data
Table 2 Main clinical characteristics and results of the tests performed for detection of coronary abnormalities

\begin{tabular}{|c|c|}
\hline & Total, $n=37$ \\
\hline Male sex, $n(\%)$ & $22(59 \%)$ \\
\hline Age at exam (year) & $13.6 \pm 3$ \\
\hline BSA & $1.4 \pm 0.5$ \\
\hline \multicolumn{2}{|l|}{ Indications } \\
\hline Transition to adult clinic & $7(19 \%)$ \\
\hline Chest pain/clinical deterioration & $7(19 \%)$ \\
\hline Follow-up & $13(35 \%)$ \\
\hline Coronary disease previously found at cath/MRI & $10(27 \%)$ \\
\hline \multicolumn{2}{|l|}{ Diagnosis } \\
\hline TGS S/P ASO & $9(24 \%)$ \\
\hline Kawasaki disease & $7(19 \%)$ \\
\hline Heart transplant & $11(30 \%)$ \\
\hline Congenital coronary abnormality & $9(24 \%)$ \\
\hline Other & $1(3 \%)$ \\
\hline Patient on medication, $n(\%)$ & $2(6 \%)$ \\
\hline Beta blockers & $0(0 \%)$ \\
\hline Calcium channel blockers & $0(0 \%)$ \\
\hline Ace inhibitor & $2(6 \%)$ \\
\hline Termination due to muscular exhaustion, $n(\%)$ & $37(100 \%)$ \\
\hline ECG abnormalities during stress, $n(\%)$ & $3(8 \%)$ \\
\hline Arrhythmia, $n(\%)$ & $0(0 \%)$ \\
\hline Symptoms & $1(3 \%)$ \\
\hline Rest HR (bpm) & $87 \pm 12.2$ \\
\hline Peak HR (bpm) & $155.7 \pm 17.9$ \\
\hline Coronary angiograms available, $n(\%)$ & $20(54 \%)$ \\
\hline Cardiac MRI available, $n(\%)$ & $21(57 \%)$ \\
\hline Coronary angio or MRI available & $26(70 \%)$ \\
\hline
\end{tabular}

of the subjects performing the 50 tests are summarized in Table 1.

Feasibility and Safety

ESE was successfully performed and well tolerated by all patients. Satisfactory images were obtained in the entire cohort, and no patient was excluded due to the poor image quality of the scan. No significant arrhythmia (sustained atrial or ventricular tachycardia) or complication was detected, and all the studies were interrupted for muscular exhaustion. We recorded only a few isolated ventricular ectopic beats in one patient, one patient reported feeling of dizziness, and another patient presented with chest discomfort. In five patients (10\% of the studies), ST depressions were noted on ECG during exercise in absence of symptoms. Two of these patients were subjects with $\mathrm{HCM}$, the remaining three were subjects with suspected $\mathrm{CAD}$, and only in one case, the ST depression was 
Table 3 Systematic description of the nine cases with evidence of RWMA during ESE +positive, -negative, /not performed, $C C A$ congenital coronary abnormality, $T G A$ transposition of the great arteries, $A S O$ arterial switch operation, HTx heart transplant

\begin{tabular}{llllllll}
\hline Patient & Diagnosis & $\begin{array}{l}\text { RWMA } \\
\text { at rest }\end{array}$ & $\begin{array}{l}\text { RWMA at } \\
\text { low } \\
\text { exercise }\end{array}$ & $\begin{array}{l}\text { RWMA at } \\
\text { peak } \\
\text { exercise }\end{array}$ & $\begin{array}{l}\text { RWMA } \\
\text { at } \\
\text { recovery }\end{array}$ & $\begin{array}{l}\text { Coronary } \\
\text { stenosis }>70 \% \\
\text { at cath }\end{array}$ & $\begin{array}{l}\text { LG } \\
\text { enhancement } \\
\text { at MRI }\end{array}$ \\
\hline 1 & $\begin{array}{l}\text { TGA S/P } \\
\text { ASO }\end{array}$ & + & + & - & + & - & - \\
2 & HTx & + & - & - & + & + & $/$ \\
3 & CCA & - & + & + & - & $/$ & $/$ \\
4 & HTx & + & + & - & + & - & + \\
5 & HTx & + & + & + & + & + & + \\
6 & HTx & + & + & + & + & + & - \\
7 & CCA & + & + & + & + & - & - \\
8 & TGA S/P & + & + & + & + & - & - \\
9 & ASO & & & & & + & +
\end{tabular}

associated to the presence of RWMA. Resting HR was $82 \pm 13$, and peak HR was $153 \pm 19(81 \pm 15 ; 159 \pm 17$ excluding heart transplant patients and patients on beta blockers or calcium channel blockers). The main results of all the tests performed are summarized in Table 2 .

\section{Coronary Artery Disease Analysis}

Thirty-seven exams (mean age at the time of the exam $13.6 \pm 3$ ) were performed for detection of CAD in 29 patients. Nine $(24 \%)$ exams were performed in patients who had undergone repair of congenital coronary abnormalities (five patients with anomalous left coronary artery from the pulmonary artery (ALCAPA), two patients with right coronary artery from the left coronary sinus, one patient with left coronary artery from the right coronary sinus with intramural course, one patient with left main coronary artery occlusion of unknown origin), 11 (30\%) in heart transplant recipients, 7 (19\%) in patients with coronary artery abnormalities after Kawasaki disease, and 9 (24\%) in patients who had previously undergone arterial switch operation for transposition of the great arteries. One (3\%) patient was referred for typical chest pain after surgical repair for double outlet right ventricle. The indication for the test was chest pain or clinical deterioration in 7 (19\%) cases, clinical follow-up in 13 (35\%), CAD previously found at coronary angiography or MRI in 10 $(27 \%)$ and in $7(19 \%)$ cases the test was performed as part of the routine protocol in our institution prior to transition to adult services. One patient reported mild dizziness during recovery. Resting HR was $87 \pm 12.2$, and peak HR was $155.7 \pm 17.0$ (Table 2 ).

Nine patients were identified to have RWMA. A complete description of these nine cases and the comparison with coronary angiography and cardiac MRI (when available) are shown in Table 3 . In the remaining 28 tests
Table 4 Main clinical characteristics and results of the tests performed in patients with $\mathrm{HCM}$

\begin{tabular}{ll}
\hline & Total, $n=10$ \\
\hline Male sex, $n(\%)$ & $5(50 \%)$ \\
Age at exam (year) & $14.6 \pm 3.6$ \\
BSA & $1.3 \pm 0.5$ \\
Indications & \\
Exertional chest pain & $7(70 \%)$ \\
Exertional presyncope & $2(20 \%)$ \\
Exercise-induced fatigue & $1(10 \%)$ \\
Medication & $9(90 \%)$ \\
Beta blocker & $7(77 \%)$ \\
CCBs & $4(44 \%)$ \\
Termination due to muscular exhaustion, $n(\%)$ & $10(100 \%)$ \\
ECG abnormalities during stress, $n(\%)$ & $2(20 \%)$ \\
Arrhythmia, $n(\%)$ & $0(0 \%)$ \\
Symptoms & $1(10 \%)$ \\
Resting HR (bpm) & $74.1 \pm 14.2$ \\
Peak HR (bpm) & $134.3 \pm 14.9$ \\
LVOT gradient at rest, mmHg & $22.5 \pm 11.2$ \\
LVOT gradient at peak exercise, mmHg & $39.8 \pm 30.5$ \\
LVOT gradient at rest $\geq 30 \mathrm{mmHg}, n(\%)$ & $2(20 \%)$ \\
LVOT gradient $\geq 30$ mmHg at peak exercise, $n(\%)$ & $5(50 \%)$ \\
\hline
\end{tabular}

CCBs calcium channel blockers, LVOT left ventricle outflow tract

without any evidence of RWMA on ESE, coronary angiography or cardiac MRI when available demonstrated significant coronary stenosis in four cases $(14 \%)$.

Hypertrophic Cardiomyopathy Population

Ten patients affected by HCM (mean age $14.6 \pm 3.6$ ) were studied. No significant arrhythmia was encountered. The indication for ESE in all cases was the clinical suspicion of 
provocable LV outflow tract obstruction in the absence of a resting gradient on transthoracic echocardiography. Seven patients $(70 \%)$ had exertional chest pain, two $(20 \%)$ presented with presyncope on exercise, and one patient (10\%) had increasing exercise-related fatigue. Only one patient reported symptoms during the test (mild chest discomfort). Nine patients (90\%) were on medications (7 on beta blockers, 4 on calcium channel blockers, and 3 on both). Resting HR was $74.1 \pm 14.2$, and peak HR was $134.3 \pm 14.9$. Mean resting left ventricular outflow tract (LVOT) gradient was $22.5 \pm 11.2$, and peak LVOT gradient was $39.8 \pm 30.5$. LVOT gradient was $\geq 30 \mathrm{mmHg}$ at rest in two patients $(20 \%)$, and 3 new patients $(30 \%)$ developed a LVOT gradient $\geq 30 \mathrm{mmHg}$ during exercise. The main data of ESE in HCM population are summarized in Table 4.

\section{Image Quality and Interobserver Variability}

Image quality and the interobserver variability were analyzed in 29 patients (1 exam for each patient assessed for potential coronary artery disease). Four hundred and sixty four different views were analyzed. $403(87 \%)$ views were judged as optimal (score $=1), 32(7 \%)$ as suboptimal ( score $=2)$, and $29(6 \%)$ as inadequate (score $=3)$. The view more inclined to be suboptimal was the two-chamber view for assessing the anterior and inferior LV wall.

The coefficient of agreement $(K)$ among the two investigators for the analysis of image quality was 0.7276 (95\% CI 0.6497-0.8055), representing a good correlation. For the wall motion analysis (2329 segments analyzed), the $K$ was 0.5125 (95\%CI $0.4782-0.5468)$, representing a moderate agreement between the two observers.

\section{Discussion}

Stress echocardiography in children is not as well established as a diagnostic modality as it is in the adult population. The scarce data available in this setting, characterized by a wide heterogeneity in terms of characteristics of the studied populations and type of stressor, have very likely contributed to the lack of a shared protocol in the pediatric community. Our study shows that bicycle stress echocardiography performed by on-line scanning during exercise is a feasible, well-tolerated, and reproducible modality in children, and our protocol could be safely applied by other specialists in pediatric echocardiography.

The wide heterogeneity in terms of the studied cohort and indications for testing is testified by the presence of isolated reports on use of stress echocardiography in children in completely different clinical settings. There are few reports focused on assessing the presence of CAD after heart transplantation $[12,15,16,28,30,46]$ or after arterial switch operation in patients born with transposition of the great arteries [19, 23] or in subjects with history of Kawasaki disease [20, 24, 34, 47], as well as studies providing dynamic evaluation in patients with congenital left heart disease [1, 5, 14, 35], repaired tetralogy of Fallot [3, 10], repaired complex congenital heart defects [19, 26, 38], and after Fontan operation [9].

The un-standardized nature of this investigation is demonstrated further by the number of different stressor agents used. Dobutamine, adenosine, and dipyridamole have been used as pharmacological stressors, and both treadmill and bicycle exercise testing have been utilized when ESE. This large heterogeneity in terms of the background of the patients and type of stressor has not encouraged the establishment of an accepted stress echocardiography protocol in children, and may, at least in part, explain the conflicting results in terms of sensitivity and specificity obtained by the different studies assessing children with CAD [12, 19, 23, 46].

The rapid drop of heart rate in children just after the peak exercise phase [36] may be a further contributing factor to these conflicting results. The latter has previously been identified as the most important limitation of the technique, with a rapid return to resting heart rate after stopping exercise, which can occur within $1 \mathrm{~min}$ in some children. This limitation is particularly important when the standard treadmill exercise protocol is used, forcing the acquisition of the images after the end of the exercise. In our study, the wide difference between HR at rest and peak $\mathrm{HR} \quad(82 \pm 13.6$ at rest, $153.4 \pm 19.7$ at peak; $81.3 \pm 15-158.9 \pm 17.1$ excluding heart transplant and patients on beta blockers or calcium channel blockers) testifies that bicycle ESE allowing on-line scanning may resolve this technical problem in children.

Image quality is a well-known technical limitation in exercise stress echocardiography [12], so that the use of contrast agents has been also proposed in children [27] to improve LV border delineation. In our experience, using a semi-supine bicycle ergometer, the images were obtained in satisfactory quality in the entire cohort of patients, and none of them was excluded due to the poor image quality of the scan. The systematic analysis of all images confirmed very high quality in more than $85 \%$.

Concerns have also been raised about the safety of the technique in children, with one report outlining a high prevalence of side effects, including hypertension, arrhythmia, and chest pain during dobutamine stress exercise in children [3]. In our cohort, we experienced mild adverse effects in less than $5 \%$ of patients and no significant arrhythmia.

Very few data are available on reliability of stress echocardiography [31]. To our knowledge, interobserver 
variability of ESE in children has never been assessed. We tested interobserver variability for image quality of the acquired views, demonstrating a good agreement between the two different observers. A slightly worse but anyway moderately good agreement was found in the grading of regional wall motion abnormalities. Our analysis on grading of RWMA was performed comparing each LV segments analyzed for all the views obtained. This extensive and complete analysis very likely imposed a handicap on the result, and the agreement would have likely been better if the analysis had compared just the presence of RWMA. However, this result might suggest that ESE for assessing of RWMA in children is not a straightforward technique, and should be performed by experienced operators who are properly trained.

We have also demonstrated that ESE may be a valuable technique in children with HCM. A significant number of patients without resting LV outflow tract gradients may develop dynamic obstruction with exercise, and detection of an exercise-induced gradient may provide a therapeutic target for the relief of symptoms as well as enabling more reliable monitoring of the response to therapy [4]. To the best of our knowledge, this is the first study assessing the feasibility of ESE in this setting in the pediatric population, and further, larger studies are warranted to optimize the technique and assess its clinical utility.

\section{Limitations}

Our study did not explicitly focus on ESE diagnostic accuracy of detection of coronary stenosis in children. Although the results comparing ESE with coronary angiography and cardiac MRI were presented, the limited number of patients with simultaneous coronary angiography or cardiac MRI did not allow any statistical analysis to be performed. Even the rate of false positive and false negative in our study must be reassessed in a dedicated prospective study about ESE diagnostic effectiveness. However, the latter could be partially explained by the presence of extensive collateral circulation in children, while it is well known that a sizable proportion (more than $30 \%$ ) of patients with positive ESE has no significant coronary lesion in the adult population [18]. Nonetheless in a large adult cohort, outcomes of patients with false-positive results were similar to those of patients with true-positive results, suggesting a prognostic value of the ESE even in absence of CAD, and suggesting that patients with false-positive results on ESE should still receive intensive risk factor management and careful clinical follow-up [18].

\section{Conclusions}

Exercise stress echocardiography is a feasible, safe, and reproducible modality in children. Exercise performed on a semi-supine bicycle ergometer allows acquisition of good quality on-line images and avoids the rapid drop of heart rate after exercise in children representing an ideal type of stressor in pediatric population. Further data are needed to assess its diagnostic accuracy in assessing for the presence of $\mathrm{CAD}$, and its possible role in risk and prognostic stratification in the pediatric setting.

In children with HCM, bicycle stress echocardiography can unmask latent LV outflow tract dynamic obstruction during exercise in patients without a resting gradient.

\section{Conflict of interest None.}

Open Access This article is distributed under the terms of the Creative Commons Attribution License which permits any use, distribution, and reproduction in any medium, provided the original author(s) and the source are credited.

\section{References}

1. Alpert BS, Bloom KR, Olley PM (1980) Assessment of left ventricular contractility during supine exercise in children with left-sided cardiac disease. Br Heart J 44:703-710

2. Altman DG (1991) Statistics in medical journals: developments in the 1980s. Stat Med 10:1897-1913

3. Apostolopoulou SC, Laskari CV, Tsoutsinos A, Rammos S (2007) Doppler tissue imaging evaluation of right ventricular function at rest and during dobutamine infusion in patients after repair of tetralogy of Fallot. Int J Cardiovasc Imaging 23:25-31

4. Argulian E, Chaudhry FA (2012) Stress testing in patients with hypertrophic cardiomyopathy. Prog cardiovasc Dis 54:477-482 2012/06/13

5. Banaszak P, Szkutnik M, Kusa J, Banaszak B, Bialkowski J (2009) Utility of the dobutamine stress echocardiography in the evaluation of the effects of a surgical repair of aortic coarctation in children. Cardiol J 16:20-25

6. Bombardini T, Gherardi S, Arpesella G, Maccherini M, Serra W, Magnani G et al (2011) Favorable short-term outcome of transplanted hearts selected from marginal donors by pharmacological stress echocardiography. J Soc Echocardiogr 24:353-362

7. Bonow RO, Carabello BA, Kanu C et al (2006) ACC/AHA 2006 guidelines for the management of patients with valvular heart disease: a report of the American College of Cardiology/American Heart Association Task Force on Practice Guidelines (writing committee to revise the 1998 Guidelines for the Management of Patients With Valvular Heart Disease): developed in collaboration with the Society of Cardiovascular Anesthesiologists: endorsed by the Society for Cardiovascular Angiography and Interventions and the Society of Thoracic Surgeons. Circulation 114:e84-e231

8. Bossone E, Rubenfire M, Bach DS, Ricciardi M, Armstrong WF (1999) Range of tricuspid regurgitation velocity at rest and during exercise in normal adult men: implications for the diagnosis of pulmonary hypertension. J Am Coll Cardiol 33:1662-1666

9. Brili SV, Alexopoulos NA, Barberis VI et al (2007) Dobutamine stress echocardiography for the evaluation of cardiac reserve late after Fontan operation. Hell J Cardiol 48:252-257

10. Brili S, Stamatopoulos I, Barbetseas J et al (2008) Usefulness of dobutamine stress echocardiography with Tissue Doppler imaging for the evaluation and follow-up of patients with repaired tetralogy of Fallot. J Am Soc Echocardiogr 21:1093-1098 
11. Brothers JA, McBride MG, Seliem MA et al (2007) Evaluation of myocardial ischemia after surgical repair of anomalous aortic origin of a coronary artery in a series of pediatric patients. J Am Coll Cardiol 50:2078-2082

12. Chen MH, Abernathey E, Lunze F et al (2012) Utility of exercise stress echocardiography in pediatric cardiac transplant recipients: a single-center experience. J heart Lung Transplant 31:517-523

13. Cullen MW, Pellikka PA (2011) Recent advances in stress echocardiography. Curr Opin Cardiol 26:379-384

14. Cyran SE, Grzeszczak M, Kaufman K et al (1993) Aortic "recoarctation" at rest versus at exercise in children as evaluated by stress Doppler echocardiography after a "good" operative result. Am J Cardiol 71(11):963-970

15. Di Filippo S, Semiond B, Roriz R, Sassolas F, Raboisson MJ, Bozio A (2003) Non-invasive detection of coronary artery disease by dobutamine-stress echocardiography in children after heart transplantation. J Heart lung Transplant 22:876-882

16. Dipchand AI, Bharat W, Manlhiot C, Safi M, Lobach NE, McCrindle BW (2008) A prospective study of dobutamine stress echocardiography for the assessment of cardiac allograft vasculopathy in pediatric heart transplant recipients. Pediatr Transplant 12:570-576

17. Fine NM, Pellikka PA (2011) Stress echocardiography for the detection and assessment of coronary artery disease. J Nucl Cardiol 18:501-515

18. From AM, Kane G, Bruce C, Pellikka PA, Scott C, McCully RB (2010) Characteristics and outcomes of patients with abnormal stress echocardiograms and angiographically mild coronary artery disease $(<50 \%$ stenoses $)$ or normal coronary arteries. J Am Soc Echocardiogr 23:207-214

19. Hauser M, Bengel FM, Kuhn A, Sauer U, Zylla S, Braun SL et al (2001) Myocardial blood flow and flow reserve after coronary reimplantation in patients after arterial switch and ross operation. Circulation 103:1875-1880

20. Henein MY, Dinarevic S, O'Sullivan CA, Gibson DG, Shinebourne EA (1998) Exercise echocardiography in children with Kawasaki disease: ventricular long axis is selectively abnormal. Am J Cardiol 81:1356-1359

21. Himelman RB, Stulbarg M, Kircher B et al (1989) Noninvasive evaluation of pulmonary artery pressure during exercise by saline-enhanced Doppler echocardiography in chronic pulmonary disease. Circulation 79:863-871

22. Holverda S, Gan CT, Marcus JT, Postmus PE, Boonstra A, VonkNoordegraaf A (2006) Impaired stroke volume response to exercise in pulmonary arterial hypertension. J Am Coll Cardiol 47:1732-1733

23. Hui L, Chau AK, Leung MP, Chiu CS, Cheung YF (2005) Assessment of left ventricular function long term after arterial switch operation for transposition of the great arteries by dobutamine stress echocardiography. Heart 91:68-72

24. Ishii M, Himeno W, Sawa M et al (2002) Assessment of the ability of myocardial contrast echocardiography with harmonic power Doppler imaging to identify perfusion abnormalities in patients with Kawasaki disease at rest and during dipyridamole stress. Pediatr Cardiol 23:192-199

25. Jengo JA, Oren V, Conant R et al (1979) Effects of maximal exercise stress on left ventricular function in patients with coronary artery disease using first pass radionuclide angiocardiography: a rapid, noninvasive technique for determining ejection fraction and segmental wall motion. Circulation 59:60-65

26. Kaplan JD, Foster E, Redberg RF, Schiller NB (1994) Exercise Doppler echocardiography identifies abnormal hemodynamics in adults with congenital heart disease. Am Heart J 127:1572-1580

27. Kimball TR (2002) Pediatric stress echocardiography. Pediatr Cardiol 23:347-357
28. Kimball TR, Witt SA, Daniels SR (1997) Dobutamine stress echocardiography in the assessment of suspected myocardial ischemia in children and young adults. Am J Cardiol 79:380-384

29. Lang RM, Bierig M, Devereux RB et al (2006) Recommendations for chamber quantification. Eur J Echocardiogr 7:79-108

30. Larsen RL, Applegate PM, Dyar DA et al (1998) Dobutamine stress echocardiography for assessing coronary artery disease after transplantation in children. J Am Coll Cardiol 32:515-520

31. Leischik R, Eising EG, Katz M et al (1997) Comparison of stress echocardiography and radionuclide ventriculography in the diagnosis of cardiomyopathy in patients receiving chemotherapy for malignant diseases. Dtsch Med Wochenschr 122:1509-1515

32. Maron MS, Olivotto I, Zenovich AG et al (2006) Hypertrophic cardiomyopathy is predominantly a disease of left ventricular outflow tract obstruction. Circulation 114:2232-2239

33. Moonen M, O'Connor K, Magne J, Lancellotti P, Pierard LA (2010) Stress echocardiography for selecting potential responders to cardiac resynchronisation therapy. Heart 96:1142-1146

34. Noto N, Ayusawa M, Karasawa K et al (1996) Dobutamine stress echocardiography for detection of coronary artery stenosis in children with Kawasaki disease. J Am Coll Cardiol 27:1251-1256

35. Oyen EM, Ingerfeld G, Ignatzy K, Brode PE (1987) Dynamic exercise echocardiography in children with congenital heart disease affecting the left heart. Int J Cardiol 17:315-325

36. Pahl E, Sehgal R, Chrystof D et al (1995) Feasibility of exercise stress echocardiography for the follow-up of children with coronary involvement secondary to Kawasaki disease. Circulation 91:122-128

37. Pahl E, Duffy CE, Chaudhry FA (2000) The role of stress echocardiography in children. Echocardiography 17:507-512

38. Pauliks LB, Brian Clark J, Rogerson A, DiPietro A, Myers JL, Cyran SE (2012) Exercise stress echocardiography after childhood ross surgery: functional outcome in 26 patients from a single institution. Pediatr Cardiol 33:797-801

39. Pellikka PA, Nagueh SF, Elhendy AA, Kuehl CA, Sawada SG (2007) American Society of Echocardiography recommendations for performance, interpretation, and application of stress echocardiography. J Am Soc Echocardiogr 20:1021-1041

40. Peteiro J, Bouzas-Mosquera A, Fernandez Xet al (2012) Prognostic value of exercise echocardiography in patients with hypertrophic cardiomyopathy. J Am Soc Echocardiogr 25:182-189

41. Robbers-Visser D, Luijnenburg SE, van den Berg J, Moelker A, Helbing WA (2009) Stress imaging in congenital cardiac disease. Cardiol Young 19:552-562

42. Rocchi G, Bertini M, Biffi M et al (2009) Exercise stress echocardiography is superior to rest echocardiography in predicting left ventricular reverse remodelling and functional improvement after cardiac resynchronization therapy. Eur Heart J 30:89-97

43. Shah JS, Esteban MT, Thaman R et al (2008) Prevalence of exercise-induced left ventricular outflow tract obstruction in symptomatic patients with non-obstructive hypertrophic cardiomyopathy. Heart 94:1288-1294

44. Vahanian A, Baumgartner H, Bax J et al (2007) Guidelines on the management of valvular heart disease: The Task Force on the Management of Valvular Heart Disease of the European Society of Cardiology. Eur Heart J 28:230-268

45. Wu WC, Aziz GF, Sadaniantz A (2004) The use of stress echocardiography in the assessment of mitral valvular disease. Echocardiography 21:451-458

46. Yeung JP, Human DG, Sandor GG, De Souza AM, Potts JE (2011) Serial measurements of exercise performance in pediatric heart transplant patients using stress echocardiography. Pediatr Transplant 15:265-271

47. Zilberman MV, Goya G, Witt SA, Glascock B, Kimball TR (2003) Dobutamine stress echocardiography in the evaluation of young patients with Kawasaki disease. Pediatr Cardiol 24:338-343 